\title{
Hacia un modelo de comunicación en salud pública en América Latina y el Caribe
}

\author{
César A. Macías-Chapula ${ }^{1}$
}

Forma de citar

Macías-Chapula, CA. Hacia un modelo de comunicación en salud pública en América Latina y el Caribe. Rev Panam Salud Publica. 2005;18(6):427-38.

RESUMEN Objetivo. No existen estudios bibliométricos ni cienciométricos que permitan examinar con un criterio cuantitativo, retrospectivo e integral la producción científica sobre salud pública en América Latina y el Caribe. Además, las carencias de los sistemas de información existentes no permite examinar la pertinencia, calidad e impacto de la producción científica con miras a evaluarla en función del cumplimiento de determinadas exigencias sociales y del patrón de comunicación científica existente.

El propósito de este trabajo es presentar los resultados de un análisis bibliográfico sobre la producción científica en el área de salud pública en la Región de América Latina y el Caribe. El objetivo final del análisis es construir un modelo de comunicación científica en este campo que sirva de apoyo para que investigadores, gestores y trabajadores de la salud pública puedan tomar las decisiones y las acciones necesarias.

Método. El método utilizado consistió en una revisión bibliográfica de la base de datos LILACS-SP para identificar la distribución de las publicaciones sobre salud pública generadas por cada país en el período comprendido entre 1980 y 2002. Los datos obtenidos se procesaron con Microsoft Excel (2000) y Bibexcel (2001), para obtener indicadores de la producción científica, el tipo de documentos publicados, el idioma, el número de autores por publicación, el contenido temático y las instituciones participantes. Para efectos del presente trabajo, la muestra se limitó a los ocho países que arrojaron una producción de documentos superior a los tres mil registros durante el período de estudio. Estos países fueron, en orden descendente de cantidad de registros: Brasil, Chile, México, Argentina, Venezuela, Colombia, Perú y Cuba. En conjunto, representan $85,10 \%$ del total de la producción.

Resultados. Los datos recabados ayudaron a establecer las bases de un modelo de comunicación científica en el área de salud pública, caracterizado principalmente por los elementos señalados a continuación. Treinta y siete países participaron con 97605 documentos en el período de 1980-2002. La mayor parte de los registros aportados por esas ocho naciones correspondió a artículos publicados en revistas científicas $(67,73 \%)$ y a monografías $(29,46 \%)$. La proporción de trabajos firmados por dos o más autores fue relativamente alta, de 56,48\% entre los registros analizados. La producción correspondiente a artículos científicos (56 253) se distribuyó entre 929 diferentes títulos de revistas. Las revistas con mayor producción, en orden descendente, fueron: Revista de Saúde Pública; Cadernos de Saúde Pública, Revista Médica de Chile, Archivos Latinoamericanos de Nutrición y Salud Pública de México. La clasificación temática de las revistas participantes se distribuyó en 29 diferentes especialidades, principalmente de las áreas de medicina general y pediatría. La producción consiste principalmente en estudios en seres humanos en general $y$, en orden descendente, de sexos femenino y masculino y de edad adulta; en menor proporción, en embarazadas y personas de edad mediana o ancianos. En el ámbito regional, el contenido de la producción coincide en aspectos como factores de riesgo, políticas de salud y atención primaria de la salud. Se identifica

1 Dirección de Investigación, Hospital General de México, México D.F. Toda correspondencia deberá diri- girse a: César A. Macías-Chapula, Algeciras 43 A, Col. Insurgentes Mixcoac, Del. Benito Juárez, México,
D.F. 03920. Teléfono y fax: 10350608 y 599961 33, ext.1235. Correo electrónico: chapula@data.net.mx 
una preponderancia de ciertas áreas temáticas entre los países participantes, como el de los médicos de familia en Cuba.

Conclusiones. Se obtuvo un modelo preliminar de comunicación en salud pública en América Latina y el Caribe que se espera ayude a sentar las bases para una mayor investigación orientada hacia el desarrollo de un modelo de comunicación científica en el área.

Palabras clave Salud pública; América Latina; Región del Caribe; Brasil; Chile; México; Argentina; Venezuela; Colombia; Perú; Cuba.

Una clasificación aceptada de la investigación en el campo de la salud (1) define la investigación biomédica como el estudio de las condiciones de salud del individuo, la investigación clínica como el estudio de las respuestas individuales y la investigación en salud pública como el estudio de las condiciones y respuestas en el ámbito poblacional. Parece conveniente el énfasis que establece esta clasificación tanto en los objetivos (condiciones y respuestas) como en los diferentes niveles de análisis (el individuo y la población).

Por otro lado, dentro de un sistema de salud, la investigación es tan importante como el financiamiento (2). Efectivamente, los resultados de la investigación no solo representan el conocimiento generado o modificado por los investigadores, sino que también constituyen un producto que tiene valor en el mercado y que puede utilizarse como insumo para transformar la realidad, tomar decisiones o resolver problemas. En diciembre del año 2000, la Comisión sobre Macroeconomía y Salud de la Organización Mundial de la Salud (OMS) destacó que mediante la inversión en salud e investigación se podrían salvar las vidas de 8 millones de personas para el año 2010 (3). Entonces, la actividad científica resulta fundamental para el diseño de estrategias de desarrollo y para lograr una respuesta más eficaz a las exigencias sociales. De esa manera, la gestión de la actividad científica se convierte en un elemento clave para la obtención de dichos logros.

Esta forma de concebir la investigación en el ámbito de la salud tiene implicaciones importantes para los países en vías de desarrollo, donde se han emprendido reformas a los sistemas de salud durante los dos últimos decenios, muchas de las cuales incorporan avances en los recursos y modelos de gestión (4). Sin embargo, las reformas a los sistemas de salud se están aplicando en un contexto de transiciones epidemiológicas, recursos escasos y recortes importantes del presupuesto (5). La situación se ve agravada por las presiones externas relacionadas con la globalización de la economía y los cambios sociales y políticos subsiguientes. La gran dificultad de promover la investigación de salud en países en vías de desarrollo fue señalada recientemente por el Foro Global de Investigación en Salud (6). Aunque aquí se indican las políticas a seguir, este enfoque contrasta con la situación local que viven los investigadores que administran sus proyectos, quienes deben adquirir y fortalecer sus capacidades de gestión a partir de un contexto rudimentario determinado. Esta situación sigue analizándose en los foros internacionales actuales (7).

Los administradores de los recursos relacionados con la investigación en salud pública en América Latina y el Caribe, por ejemplo, no cuentan con la información necesaria para apoyar sus procesos de gestión. Es decir, no cuentan con datos que les permitan identificar las líneas de investigación de mayor o menor desarrollo; hacer evaluaciones comparativas y evaluar la visibilidad local, regional o internacional de la producción, además del impacto de los resultados en los diferentes programas de salud pública. Aun más, se desconoce la dinámica del desarrollo de la actividad científica que oriente a intervenir en los procesos de cambio y mejora social.

Por consiguiente, en América Latina y el Caribe se requiere una fuente de información que en el ámbito nacional y regional indique la situación que prevalece en materia de los resultados de la investigación en salud pública. La mayor parte de estos resultados pueden analizarse de manera cuantitativa a la luz de las publicaciones convencio- nales y no convencionales que derivan de la actividad científica en el área. Los resultados del análisis métrico de los indicadores de producción, visibilidad y colaboración, por ejemplo, conforman un recurso importante de apoyo a los procesos de toma de decisiones y acciones en materia de salud pública. La aplicación de este criterio cuantitativo se ha informado recientemente en los campos de la tuberculosis y la diabetes en la India y la China $(8,9)$ y en el campo de la salud mental en 15 países de la Organización para la Cooperación y el Desarrollo Económico (OCDE) (10).

Una revisión de la literatura sobre estudios bibliométricos relacionados con la producción científica en el área de salud que se realiza en América Latina y el Caribe reveló lo siguiente:

1. En general, los trabajos se han enfocado en estudios específicos sobre una especialidad o disciplina (1122), el análisis de una revista (2327) y estudios sobre países específi$\cos (19,23,28-33)$.

2. Se han publicado análisis bibliométricos que cubren toda la Región de América Latina y el Caribe, tocando tanto temas generales (34-36) como el aspecto del idioma $(37,38)$; asimismo, reformas a los sistemas de salud (39) y política científica (40).

3. Los trabajos relacionados con la salud pública analizan aspectos de visibilidad (presencia de las publicaciones en las bases de datos bibliográficos) (41) el desarrollo de la especialidad (42), el factor de impacto de las revistas (43) y la promoción de la investigación (44).

4. La Organización Panamericana de la Salud (OPS) ha participado activamente en la realización deproyectos, talleres y publicaciones relacionados con la producción científica en la Región (28, 45-48). El análisis de estos trabajos permite sacar en 
conclusión los siguientes tres puntos importantes: a) en la actividad científica se ha encontrado un predominio del nivel de análisis individual (biomédico y clínico) sobre el poblacional (salud pública); b) los países con mayor representatividad en la Región son Brasil, Argentina, México, Chile, Venezuela y Cuba y c) hay pruebas de la debilidad de los sistemas de información y de las estadísticas e indicadores sobre ciencia y tecnología en salud de la Región.

La revisión de la literatura ayudó a establecer que no existen estudios bibliométricos ni cienciométricos que permitan analizar con un criterio cuantitativo, retrospectivo e integral la producción científica sobre salud pública en América Latina y el Caribe. La debilidad de los sistemas de información existentes tampoco permite evaluar la pertinencia, calidad e impacto de la producción científica con miras a orientarla en función del cumplimiento de determinadas exigencias sociales (48).

La importancia de reclamar la generación de conocimiento dentro de un contexto social (49) está cobrando mayor fuerza. Así lo refleja el giro relativamente rápido en los objetivos de la investigación que se realiza en los países desarrollados, donde se está pasando de una búsqueda de la verdad hacia el objetivo más pragmático de obtener un entendimiento funcional del mundo empírico. En este contexto, el conocimiento con fuerza social es el producto de una interacción intensa y continua entre la información y otros resultados, entre la sociedad y su ambiente, entre las aplicaciones y las implicaciones de sus resultados. Se requiere entonces repensar la ciencia, para buscar un nuevo contrato social de esta con la sociedad $(49,50)$.

\section{PROPÓSITO}

El propósito de este trabajo es presentar los resultados de un análisis bibliográfico de la producción científica sobre salud pública en la Región de América Latina y el Caribe. El objetivo final del análisis es construir un modelo de comunicación científica en el área de salud pública que sirva de apoyo para que investigadores, gestores y trabajadores relacionados con esta disciplina puedan tomar las decisiones o acciones pertinentes.

\section{MÉTODOS}

Se realizó una investigación bibliográfica en la base de datos LILACS-SP (Literatura Latinoamericana en Ciencias de la Salud-Salud Pública), para identificar la distribución por países de las publicaciones generadas sobre la salud pública, correspondientes al período de 1980 a 2002. La estrategia de investigación se llevó a cabo utilizando los descriptores controlados que corresponden a la clasificación de salud pública, relacionados con la Región de América Latina y el Caribe. Con este propósito se utilizó el tesauro DeCS (Descriptores en Ciencias de la Salud) para LILACS. No se aplicaron restricciones de idioma, etiquetas generales de clasificación (check tags) ni tipo de documentos. Con este criterio, la recuperación de registros de la base de datos arrojó los trabajos que tratan el tema y el país de origen, independientemente de la aparición de estos términos en los campos del título o el resumen. El propósito de este método fue eliminar, en la medida de lo posible, errores en la recuperación de información al realizar búsquedas con palabras libres (free-text) en las diferentes secciones de los registros; por ejemplo, los campos de autor, título, resumen o institución.

Se identifican en este trabajo los siguientes factores limitantes:

1. El estudio se limitó a la base de datos LILACS-SP, la cual registra únicamente la producción regional, de manera que queda excluida del análisis la que se publica fuera de la Región y que se indiza en bases de datos reconocidas, como MEDLINE y el Science Citation Index.

2. El estudio se limitó a incluir publicaciones editadas en los países latinoamericanos y caribeños. De esa manera se excluyeron las publicaciones editadas, por ejemplo, en Estados Unidos y Europa o las pertenecientes a organismos internacionales.
Los datos se analizaron en un disco compacto que para el propósito de este estudio produjo el Centro Latinoamericano y del Caribe de Información en Ciencias de la Salud (BIREME).

Se obtuvieron subconjuntos de registros para cada uno de los países identificados en la base de datos. Estos subconjuntos se procesaron con $\mathrm{Mi}$ crosoft Excel (2000) y Bibexcel (versión 2001) a fin de obtener los datos sobre los siguientes rubros:

a) producción y distribución de la literatura durante el período de estudio

b) distribución del tipo de documentos y de la autoría

c) idioma de las publicaciones

d) contenido temático de las publicaciones

e) instituciones y países que realizaron investigación sobre la salud pública en la Región

Los registros de los documentos recuperados se clasificaron según su tipo; por ejemplo, artículos publicados en revistas científicas, libros o literatura no convencional. En el caso de artículos publicados en revistas científicas, los títulos de estas se acumularon y ordenaron en orden alfabético, para luego clasificarse en su contenido temático, conforme a la cobertura de la revista en fuentes secundarias de información.

Los datos obtenidos de los campos de autor se analizaron para identificar coautoría (colaboración). El análisis del campo correspondiente al idioma en todos los tipos de publicaciones, permitió evaluar la medida en que este contribuye a la visibilidad nacional, regional o internacional.

El análisis del contenido temático de los registros recuperados se realizó conforme a los lineamientos establecidos en la estructura del tesauro DeCS. De esta forma, se analizaron los campos de las etiquetas generales de clasificación de los artículos (check tags), los descriptores mayores y los subencabezamientos de materia encontrados.

Los datos obtenidos de los análisis se tabularon y graficaron, con el fin de representar de manera esquemática los resultados del estudio. La interpretación de los datos permitió establecer una clasificación geográfica y temá- 
tica, tanto en la escala nacional como en la regional. Este criterio permitió identificar, a manera de observatorio, el comportamiento de la producción y comunicación científica entre los países de una manera integrada, comparativa y retrospectiva.

\section{RESULTADOS}

\section{Modelo de comunicación en salud pública}

El análisis general muestra que participaron 37 países, con una producción total de 97605 documentos, correspondiente al período 1980-2002. Los resultados indicaron cuatro niveles de producción, de acuerdo con la cantidad de registros recuperados para cada uno de los países participantes. Estos niveles se clasificaron en 1) alto, 2) medio, 3) bajo y 4) mínimo.

El nivel alto correspondió a Brasil, con 41606 registros, equivalentes a $42,63 \%$ del total. El nivel medio estuvo integrado por siete países que generaron una producción de 3000 a 10000 re- gistros cada uno. Fueron los siguientes: Argentina, Chile, Colombia, Cuba, México, Perú y Venezuela. La producción de estos países fue de 41448 documentos, que representan $42,47 \%$ del total.

El nivel bajo estuvo integrado por siete países, que generaron una producción del orden de 1000 a 3000 registros cada uno. Fueron los siguientes: Bolivia, Costa Rica, Ecuador, Guatemala, Nicaragua, República Dominicana y Uruguay. La producción de estos países fue de 11105 documentos, que constituyen $11,38 \%$ del total. El nivel mínimo correspondió al resto de los 22 países incluidos en el estudio, en su mayor parte pertenecientes a las regiones de Centroamérica y el Caribe. Estos generaron menos de 1000 registros cada uno, con una producción de 3446 documentos, que representan $03,53 \%$ del total. El cuadro 1 presenta la distribución por volumen de producción, anual y acumulada, de los 37 países participantes.

En el cuadro 1 se aprecia que los primeros 15 países, con 1000 o más registros cada uno, son los que han generado una producción más regular a lo largo del período 1980-2002. Se observa también que los primeros ocho países, que representan aproximadamente la cuarta parte del total, contribuyeron con $85,10 \%$ de la producción. Estos países pertenecerían a las categorías alta y media de la clasificación que se indicó anteriormente. El análisis de este resultado indica claramente el liderazgo de estos países en la Región, si bien desde un punto de vista meramente cuantitativo de producción.

A continuación se presenta un análisis comparativo de la producción de los ocho países más productivos de la Región. El análisis detallado de la producción de cada país se ha presentado ya en otro sitio, como parte de un informe técnico sobre el tema (51).

\section{Tipo de documentos}

En general, al integrar la producción de los ocho países se encontró que la mayor parte de los registros correspondieron a artículos publicados en revistas científicas $(67,73 \%)$ y a obras monográficas $(29,46 \%)$. El cuadro 2 ilustra estos

CUADRO 1. Producción en salud pública en América Latina y el Caribe: distribución anual por país ${ }^{a}$ (LILACS-SP, 1980-2002)

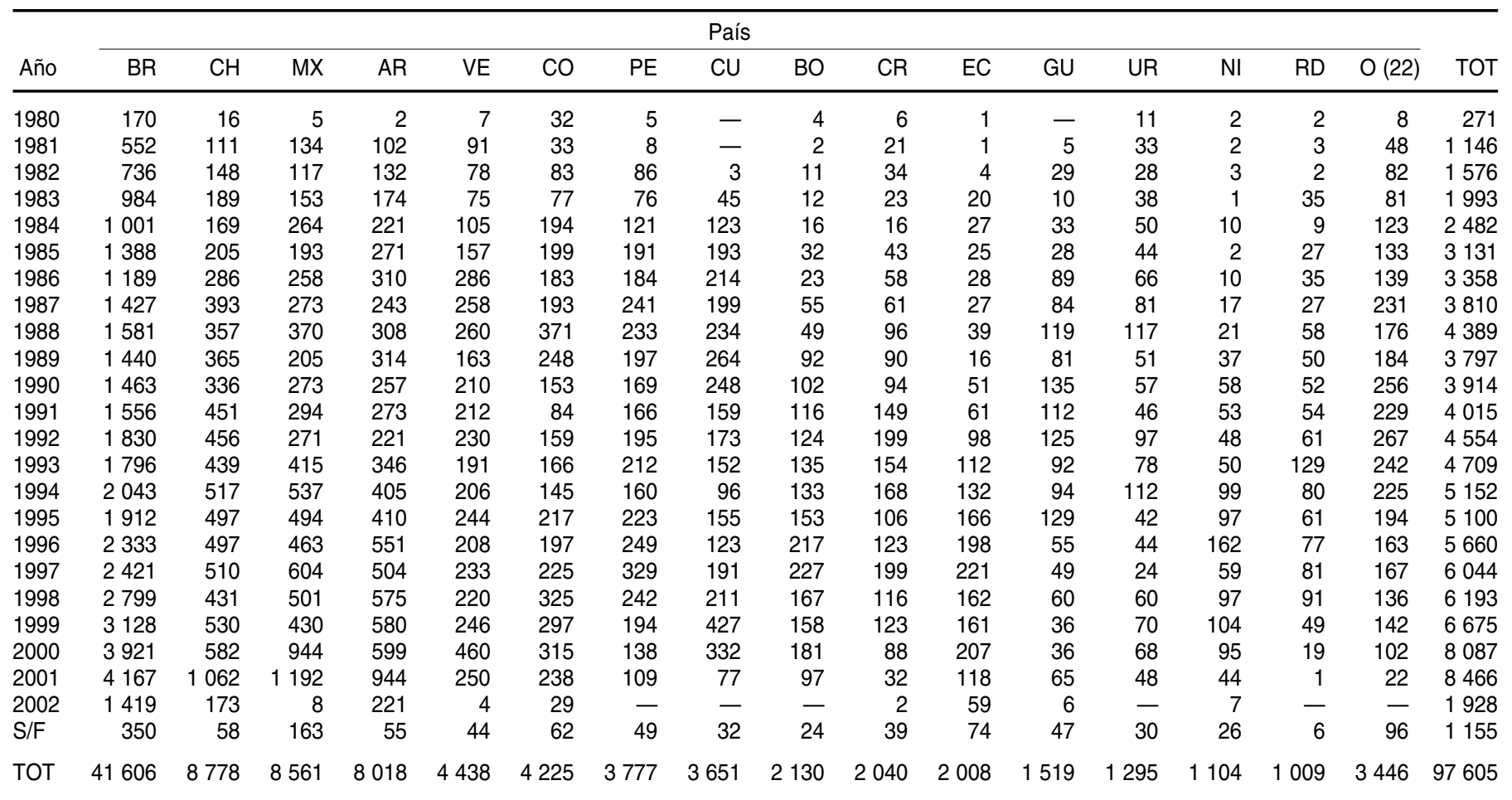

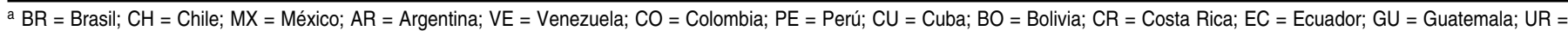
Uruguay; $\mathrm{NI}=$ Nicaragua; $\mathrm{RD}=$ República Dominicana; $\mathrm{O}(22)=$ Otros 22 países (con menos de 1000 registros cada uno) $\mathrm{S} / \mathrm{F}=\mathrm{Sin}$ fecha. 
resultados. Al realizar la comparación entre países en este aspecto, se aprecia claramente que la revista científica se utiliza como medio de comunicación en una proporción mayor en Cuba ( $88,50 \%$ de su producción), Argentina $(86,98 \%)$ y Venezuela $(82,54 \%)$ y menor en Chile (74,07\%), México $(67,74 \%)$, Brasil $(63,89 \%)$ y Colombia $(57,09 \%)$. Perú fue el único país, de los ocho analizados, que mostró un comportamiento de comunicación completamente distinto. Su mayor producción correspondió a obras monográficas $(69,02 \%)$.

\section{Autoría}

En general, la producción de documentos por un solo autor representó $43,52 \%$ del total. La proporción restante $(56,48 \%)$ se generó por la colaboración de dos o más autores. En el cuadro 3 se muestran estos resultados. En este caso destaca, por ejemplo, que Cuba registra una mayor cantidad de trabajos en colaboración que el resto de los países, dato que coincide con la alta producción de artículos en revistas que genera este país. Perú registra una mayor producción de documentos de un solo autor, lo cual se relaciona con su alta producción de obras monográficas. Esta característica pone de manifiesto que gran parte (más de 50\%) de la producción total se genera mediante trabajo en equipo, un aspecto en que destaca particularmente Cuba.

\section{Idioma}

Los documentos se publicaron en proporciones similares en español $(49,26 \%)$ y en portugués $(46,19 \%)$. El idioma inglés se encontró únicamente en $4,03 \%$ del total. Estos resultados se resumen en el cuadro 4 . La alta proporción de publicaciones en portugués coincide con la alta producción que tiene Brasil.

\section{Clasificación general de los documentos}

El análisis de la clasificación general de los artículos indica que la producción científica se orienta más hacia estudios sobre el ser humano, en orden

CUADRO 2. Producción en salud pública en ocho países latinoamericanos: distribución según el tipo de documento (LILACS-SP, 1980-2002)

\begin{tabular}{|c|c|c|c|c|c|c|c|}
\hline \multirow[b]{2}{*}{ País } & \multicolumn{2}{|c|}{ Artículos en revistas } & \multicolumn{2}{|c|}{ Monografías } & \multicolumn{2}{|c|}{ Otros } & \multirow[b]{2}{*}{ Total } \\
\hline & No. & $\%$ & No. & $\%$ & No. & $\%$ & \\
\hline Brasil & 26580 & $(63,89)$ & 13918 & $(33,45)$ & 1108 & $(02,66)$ & 41606 \\
\hline Chile & 6502 & $(74,07)$ & 2045 & $(23,30)$ & 231 & $(02,63)$ & 8778 \\
\hline México & 5799 & $(67,74)$ & 2556 & $(29,86)$ & 206 & $(02,40)$ & 8561 \\
\hline Argentina & 6974 & $(86,98)$ & 891 & $(11,11)$ & 153 & $(01,91)$ & 8018 \\
\hline Venezuela & 3663 & $(82,54)$ & 618 & $(13,92)$ & 157 & $(03,54)$ & 4438 \\
\hline Colombia & 2412 & $(57,09)$ & 1537 & $(36,38)$ & 276 & $(06,53)$ & 4225 \\
\hline Perú & 1092 & $(28,91)$ & 2607 & $(69,02)$ & 78 & $(02,07)$ & 3777 \\
\hline Cuba & 3231 & $(88,50)$ & 316 & $(08,66)$ & 104 & $(02,84)$ & 3651 \\
\hline Total & 56253 & $(67,73)$ & 24488 & $(29,48)$ & 2313 & $(02,79)$ & 83054 \\
\hline
\end{tabular}

CUADRO 3. Producción en salud pública en ocho países latinoamericanos: distribución del número de documentos según el número de autores (LILACS-SP, 1980-2002)

\begin{tabular}{|c|c|c|c|c|c|c|c|}
\hline \multirow[b]{2}{*}{ País } & \multicolumn{2}{|c|}{1 autor } & \multicolumn{2}{|c|}{2 a 4 autores } & \multicolumn{2}{|c|}{5 o más autores } & \multirow[b]{2}{*}{ Total } \\
\hline & No. & $\%$ & No. & $\%$ & No. & $\%$ & \\
\hline Brasil & 17490 & $(42,04)$ & 17839 & $(42,88)$ & 6277 & $(15,08)$ & 41606 \\
\hline Chile & 4121 & $(46,95)$ & 3365 & $(38,33)$ & 1292 & $(14,72)$ & 8778 \\
\hline México & 3353 & $(39,17)$ & 3778 & $(44,13)$ & 1430 & $(16,70)$ & 8561 \\
\hline Argentina & 4352 & $(54,28)$ & 2440 & $(30,43)$ & 1226 & $(15,29)$ & 8018 \\
\hline Venezuela & 1560 & $(35,15)$ & 2039 & $(45,94)$ & 839 & $(18,91)$ & 4438 \\
\hline Colombia & 2122 & $(50,22)$ & 1689 & $(39,98)$ & 414 & $(09,80)$ & 4225 \\
\hline Perú & 2250 & $(59,57)$ & 1253 & $(33,18)$ & 274 & $(07,25)$ & 3777 \\
\hline Cuba & 893 & $(24,46)$ & 2089 & $(57,22)$ & 669 & $(18,32)$ & 3651 \\
\hline Total & 36141 & $(43,52)$ & 34492 & $(41,53)$ & 12421 & $(14,95)$ & 83054 \\
\hline
\end{tabular}

CUADRO 4. Producción en salud pública en ocho países latinoamericanos: distribución de documentos según el idioma (LILACS-SP, 1982-2002)

\begin{tabular}{|c|c|c|c|c|c|c|c|c|}
\hline \multirow[b]{2}{*}{ País } & \multicolumn{2}{|c|}{ Español } & \multicolumn{2}{|c|}{ Portugués } & \multicolumn{2}{|c|}{ Inglés } & Otro & \multirow[b]{2}{*}{ Total } \\
\hline & No. & $\%$ & No. & $\%$ & No. & $\%$ & No. \% & \\
\hline Brasil & \multicolumn{2}{|c|}{$594(01,43)$} & \multicolumn{2}{|c|}{$38187(91,78)$} & \multicolumn{2}{|c|}{$2523(06,06)$} & $302(0,73)$ & 41606 \\
\hline Chile & \multicolumn{2}{|c|}{$8491(96,73)$} & \multicolumn{2}{|c|}{$36(0,41)$} & \multicolumn{2}{|c|}{$230(02,62)$} & $21(0,24)$ & 8778 \\
\hline México & \multicolumn{2}{|c|}{$8310(97,07)$} & \multicolumn{2}{|c|}{$13(0,15)$} & \multicolumn{2}{|c|}{$195(02,28)$} & $43(0,50)$ & 8561 \\
\hline Argentina & \multicolumn{2}{|c|}{$7850(97,91)$} & \multicolumn{2}{|c|}{$30(0,37)$} & \multicolumn{2}{|c|}{$120(01,50)$} & $18(0,22)$ & 8018 \\
\hline Venezuela & \multicolumn{2}{|c|}{$4181(94,21)$} & \multicolumn{2}{|c|}{$71(01,60)$} & \multicolumn{2}{|c|}{$182(04,10)$} & $4(0,09)$ & 4438 \\
\hline Colombia & \multicolumn{2}{|c|}{$4190(99,17)$} & \multicolumn{2}{|c|}{$7(0,17)$} & \multicolumn{2}{|c|}{$22(0,52)$} & $6(0,14)$ & 4225 \\
\hline Perú & \multicolumn{2}{|c|}{$3659(96,88)$} & \multicolumn{2}{|c|}{$13(0,34)$} & \multicolumn{2}{|c|}{$72(01,91)$} & $33(0,87)$ & 3777 \\
\hline Cuba & \multicolumn{2}{|c|}{$3640(99,70)$} & \multicolumn{2}{|c|}{$1(0,03)$} & \multicolumn{2}{|c|}{$6(0,16)$} & $4(0,11)$ & 3651 \\
\hline Total & \multicolumn{2}{|c|}{$40915(49,26)$} & $3835 \varepsilon$ & $(46,19)$ & 3350 & $(04,03)$ & $431(0,52)$ & 83054 \\
\hline
\end{tabular}

descendente, del sexo femenino, del sexo masculino y de edad adulta. Los estudios en niños y adolescentes ocuparon un lugar entre mediano y alto. Sin embargo, se encontró que, con excepción de México, los estudios sobre personas de edad mediana no ocuparon un lugar alto en la producción. Por otro lado, los estudios sobre ancianos ocuparon un lugar medio en México, Argentina y Cuba, mientras que los demás países ocupan un lugar bajo.
Los estudios sobre animales ocuparon un lugar mediano solamente en Brasil, y bajo en otros países. Los informes de casos ocuparon un lugar alto en Venezuela y Colombia. Por último, los estudios sobre el embarazo ocuparon lugares bajos en la producción de todos los países. El cuadro 5 ilustra la comparación de contenidos generales de los ocho países, según el lugar que ocuparon en la distribución de los registros. 
CUADRO 5. Producción en salud pública en ocho países latinoamericanos: clasificación general (check-tags) de los documentos, distribuidos por nivel producción y país (LILACSSP, 1980-2002)

\begin{tabular}{|c|c|c|c|c|c|c|c|c|}
\hline $\begin{array}{c}\text { Clasificación } \\
\text { temática }\end{array}$ & Brasil & Chile & México & Argentina & Venezuela & Colombia & Perú & Cuba \\
\hline Humano & 1 & 1 & 1 & 1 & 1 & 1 & 1 & 1 \\
\hline Femenino & 2 & 2 & 2 & 2 & 2 & 2 & 2 & 2 \\
\hline Masculino & 3 & 3 & 3 & 3 & 3 & 3 & 3 & 3 \\
\hline Adulto & 4 & 4 & 4 & 4 & 7 & 4 & 4 & 4 \\
\hline Niño & 5 & 5 & 7 & 5 & 6 & 6 & 6 & 7 \\
\hline Adolescencia & 6 & 6 & 6 & 7 & 8 & 7 & 5 & 5 \\
\hline Edad mediana & 7 & 7 & 5 & 6 & 11 & 15 & 8 & 6 \\
\hline Animal & 8 & 14 & 14 & 15 & 12 & 16 & 16 & 13 \\
\hline Menor de un año & 9 & 9 & 10 & 10 & 9 & 8 & 10 & 9 \\
\hline Informe de casos & 10 & 12 & 9 & 9 & 5 & 5 & 7 & 15 \\
\hline Lactante & 11 & 8 & 11 & 12 & 10 & 9 & 12 & 10 \\
\hline Anciano & 12 & 10 & 8 & 8 & 15 & 14 & 11 & 8 \\
\hline Recién nacido & 13 & 11 & 13 & 11 & 14 & 11 & 9 & 11 \\
\hline Est. compar. & 14 & 15 & 12 & 14 & 4 & 13 & 13 & 14 \\
\hline Embarazo & 15 & 13 & 15 & 13 & 13 & 10 & 14 & 12 \\
\hline
\end{tabular}

Est. comprar. $=$ Estudio comparativo.

CUADRO 6. Producción en salud pública en ocho países latinoamericanos: distribución del total de revistas publicadas (LILACSSP, 1980-2002)

\begin{tabular}{lrr}
\hline & \multicolumn{2}{c}{ Revistas } \\
\cline { 2 - 3 } País & No. & $\%$ \\
\hline Brasil & 392 & 42,19 \\
Chile & 93 & 10,01 \\
México & 71 & 07,64 \\
Argentina & 157 & 16,90 \\
Venezuela & 81 & 08,72 \\
Colombia & 60 & 06,46 \\
Perú & 35 & 03,77 \\
Cuba & 40 & 04,31 \\
$\quad$ Total & 929 & 100 \\
\hline
\end{tabular}

\section{Revistas científicas}

La producción de los ocho países en el renglón de artículos publicados en revistas científicas (56 253) se realizó en un total de 929 revistas diferentes. La distribución de estas revistas, por país, se ilustra en el cuadro 6. En este aspecto se aprecia una distribución similar a la observada en la producción total por país (cuadro 1). Una excepción importante es Argentina, país que registra mayor cantidad de revistas que Chile y México.

Al efectuar un análisis de las revistas que generaron 150 o más artículos en el período de estudio, se encontró un núcleo básico de 91 revistas de siete países (solo Perú no contó con revistas que generaran más de 150 artículos). Estas revistas contribuyeron con 26467 artículos científicos, equivalentes a $47,05 \%$ del total de la producción. Las 10 revistas con mayor producción fueron, en orden descendente, las siguientes: Revista de Saúde Pública (Brasil), Cadernos de Saúde Pública (Brasil), Revista Médica de Chile (Chile), Archivos Latinoamericanos de Nutrición (Venezuela), Salud Pública de México (México), Jornal de Pediatría (Brasil), Revista Brasileira de Enfermagem (Brasil), Gaceta Médica de México (México), Revista Chilena de Pediatría (Chile) y Revista Médica del IMSS (México).

Al evaluar la clasificación temática de las revistas participantes se encontraron 29 especialidades diferentes. En orden descendente, la clasificación mostró la distribución siguiente: medicina general (18 revistas), pediatría (10), enfermería (8), ginecología y obstetricia (7), salud pública (7), nutrición (4) y psiquiatría (4). Otras 22 especialidades aportaron tres o menos revistas cada una. En el anexo 1 se enumeran las revistas con 150 o más artículos, distribuidas por nivel de producción, país de origen y clasificación temática. Este resultado muestra claramente la amplia dispersión que tiene entre las diferentes revistas científicas la producción de textos en este campo. Además, pone de manifiesto el núcleo básico de Bradford $(52,53)$, que integra los títulos con mayor producción en salud pública.

\section{Contenidos temáticos específicos}

El análisis de los 25 descriptores de mayor frecuencia en cada país permitió encontrar el siguiente patrón de contenido de los artículos analizados: El descriptor de "factores de riesgo" resultó ser el único tema de alto interés para los ocho países analizados. Otros descriptores relevantes que compartieron la mayoría de los países fueron los siguientes: investigación, factores socioeconómicos, política de salud, atención primaria de salud, salud pública, participación comunitaria, congresos, estado nutricional, morbilidad y mortalidad infantil.

Otros descriptores identificaron áreas de interés nacional, como es el caso de: enfermería y política social en Brasil; desarrollo infantil en Chile; estudios epidemiológicos y manuales en México; odontólogos, médicos y neoplasias en Argentina; medicina, valor nutritivo y dieta en Venezuela; derechos humanos en Colombia; salud ambiental y altitud en Perú, y médicos de familia en Cuba. El anexo 2 ofrece una lista de los 25 descriptores que generaron una mayor cantidad de registros para cada país. Aquí es posible identificar, con un criterio cuantitativo de producción, la relevancia y comparación de los descriptores temáticos entre los ocho países analizados.

\section{DISCUSIÓN Y CONCLUSIONES}

Se obtuvo un modelo preliminar de comunicación científica en el área de salud pública, caracterizado principalmente por los siguientes elementos: 1) la mayor producción se concentra en ocho países; 2) Brasil es la nación con mayor producción de toda la Región; 3) la cantidad de documentos publicados en idioma portugués es similar a la generada en español; 4) la mayor cantidad de documentos corresponde a artículos publicados en revistas científicas; 5) la producción se realiza fundamentalmente en coautoría; 6) el trabajo en equipo se realiza principalmente en Cuba; 7) las revistas que publican artículos en el área de salud pública provienen de 29 especialidades diferentes, en particular medicina general y pediatría; (8) la producción consiste princi- 
palmente en estudios en seres humanos en general $\mathrm{y}$, en orden descendente, de sexo femenino, sexo masculino y edad adulta; en menor proporción se ocupa de embarazadas y personas de edad mediana o ancianos; (9) en el ámbito regional, el contenido de la producción coincide en temas como factores de riesgo, políticas de salud y atención primarias de la salud; (10) se identifica preponderancia de áreas temáticas específicas entre los países participantes, como en el caso de los médicos de familia en Cuba.

La investigación del modelo de comunicación científica se limita aquí al análisis cuantitativo de la producción y al primer nivel de resolución. Es decir, identifica apenas el marco general de la distribución de los registros (salidas) del sistema de producción científica, tanto en tiempo (1980-2002) como en tipo de producción (artículos de revistas, tesis, etc.) y en contenido (descriptores mayores y subencabezamientos). Estos elementos permitieron elaborar un esquema comparativo entre los países, encaminado a identificar fortalezas y debilidades del sistema, con un criterio descriptivo y en la perspectiva que puede ofrecer el uso de la base de datos LILACS.

Es necesario impulsar el modelo hacia un segundo y tercer nivel de resolución, donde puedan identificarse, por ejemplo, cambios en los patrones de producción de contenidos en el transcurso del tiempo. Existen dos áreas particularmente interesantes; la primera, referente a los grupos de edad, permitirá identificar a los países que continúan produciendo una mayor cantidad de documentos sobre estudios en niños y, por otra parte, a aquellos que incrementan paulatinamente la producción de estudios relacionados con la edad avanzada y la edad mediana. Este indicador ayudaría a corroborar la transición epidemiológica de los países de la Región. La segunda área relevante se relaciona con la atención primaria de la salud. Este descriptor ya no aparece entre las 25 áreas de mayor producción de Brasil y, no obstante, sigue ocupando un lugar alto en los casos de Chile (rango 3), Cuba (rango 3) y Argentina (rango 5) y un lugar bajo en México (rango 21). El ob- servatorio de la producción y el análisis retrospectivo permitirán explorar en qué año o período surge una nueva área o cambia de posición entre los niveles de producción e interés.

También la coautoría de la producción requiere analizarse con mayor grado de resolución, para identificar si la colaboración es intra o interinstitucional, nacional o internacional, norte-sur y así sucesivamente. Es un aspecto de particular interés en países donde la colaboración en equipo es frecuente, como ocurre en Cuba, Venezuela y México. Este análisis identificaría grupos de interés en áreas específicas de trabajo.

Con respecto al idioma, surgen diversas interrogantes que conviene explorar y analizar en su oportunidad, a fin de entender la trascendencia de este factor en el modelo de comunicación científica. La fuerte presencia del idioma portugués se manifiesta no solo por la cantidad de documentos publicados en ese idioma, sino también por la variedad de revistas científicas brasileñas que se detectaron en el análisis. En este estudio se encontró que los documentos del área se publican en proporción similar en español y en portugués. Sin embargo, se desconoce la influencia que ejerce un idioma sobre el otro. No se cuenta con indicadores que señalen el uso de las publicaciones brasileñas en el resto de la Región: ¿Se leen? ¿Se citan? Y lo mismo podría decirse de la producción en español: ¿Se lee o se cita en Brasil? Un análisis de las citas que se hacen en las revistas científicas identificaría, por ejemplo, el uso de la producción de un idioma en el otro, la influencia de cada idioma y los flujos de comunicación científica en áreas específicas. Por otro lado, la baja proporción de artículos publicados en inglés $(04,03 \%)$ fue la esperada, debido al origen de los artículos fuente que se ingresan en LILACS. Sería conveniente identificar las revistas que se publican en más de un idioma, como las bilingües en español y portugués, o bien la traducción que se hace de publicaciones a cada uno de estos idiomas. Se lograría así verificar la influencia de estas acciones en el modelo de comunicación de la ciencia.

El análisis del idioma y de las citas puede complementarse con otros estu- dios acerca de la proyección que alcanza la producción de la Región en otras bases de datos convencionales, como MEDLINE, SCI, SSCI, etc.

La identificación de las revistas con mayor producción de artículos de salud pública ayudó a establecer un cuadro básico de las revistas de ocho países de la Región. Este cuadro básico, integrado por revistas en portugués y español, no solo ayudará a investigadores y grupos de interés a identificar las revistas principales de salud pública, sino que también auxiliará en los procesos de toma de decisión de bibliotecarios y académicos, al seleccionar colecciones especializadas de cada área.

Los resultados evaluados en este documento correspondieron al estudio de los ocho países que emergieron como los principales productores de información científica en salud pública en la Región. Se logró identificar el modelo de comunicación científica al primer nivel de resolución. En este contexto, los resultados del estudio contribuyeron a la obtención de indicadores de producción por país, a comparar patrones de producción entre países y a sentar las bases para seguir desarrollando el modelo a un segundo y un tercer nivel de resolución. Esta línea de trabajo ayudará a construir un observatorio de la producción y a identificar los flujos de comunicación, visibilidad e impacto de la producción, por países, en el transcurso del tiempo.

Por último, cabe advertir que no es menos importante continuar el análisis del modelo de comunicación entre los países que reportaron una producción baja y mínima (menos de tres mil documentos) en el período de 1980-2002. La integración de la producción de estos países al análisis ayudará a tener una imagen más completa del modelo de comunicación referido.

Agradecimientos. Este trabajo se realizó gracias al financiamiento del proyecto y apoyo técnico de BIREME. El autor agradece la colaboración de Alejandro Gutiérrez-Carrasco y J. Antonio Mendoza-Guerrero en la sistematización y tabulación de los datos. 


\begin{tabular}{|c|c|c|c|c|}
\hline $\begin{array}{l}\text { Nivel de } \\
\text { producción }\end{array}$ & Nombre de la revista & No. de artículos & País de origen & Clasificación temática \\
\hline 1 & Revista de Saúde Pública & 1504 & Brasil & Salud pública \\
\hline 2 & Cadernos de Saúde Pública & 1167 & Brasil & Salud pública \\
\hline 3 & Revista Médica de Chile & 935 & Chile & Medicina general \\
\hline 4 & Archivos Latinoamer de Nutr & 811 & Venezuela & Nutrición \\
\hline 5 & Salud Pública de México & 702 & México & Salud pública \\
\hline 6 & Jornal de Pediatría & 559 & Brasil & Pediatría \\
\hline 7 & Revista Brasileira de Enfermagem & 542 & Brasil & Enfermería \\
\hline 8 & Gaceta Médica de México & 500 & México & Medicina general \\
\hline 9 & Revista Chilena de Pediatría & 494 & Chile & Pediatría \\
\hline 10 & Revista Médica del IMSS & 483 & México & Medicina general \\
\hline 11 & Jornal Brasileiro de Psiquiatría & 462 & Brasil & Psiquiatría \\
\hline 12 & Bol Médico del Hosp Infant Mex & 432 & México & Pediatría \\
\hline 13 & Rev Escola Enfermagem USP & 420 & Brasil & Enfermería \\
\hline 14 & Saúde em Debate & 416 & Brasil & Medicina general \\
\hline 15 & Cuadernos Médico-Sociales & 409 & Chile & Sociología médica \\
\hline 16 & Higiene Alimentar & 395 & Brasil & Nutrición \\
\hline 17 & Memorias do Inst Oswaldo Cruz & 390 & Brasil & Salud pública \\
\hline 18 & Brazilian J Medical \& Biol Res & 352 & Brasil & Investig médica \& biológica \\
\hline 19 & Medicina (Buenos Aires) & 343 & Argentina & Medicina general \\
\hline 20 & Boletín Hosp Sn Juan de Dios & 336 & Chile & Medicina general \\
\hline 21 & Revista Chilena de Nutrición & 330 & Chile & Nutrición \\
\hline 22 & Arquivo Brasil Med Veter Zootec & 317 & Brasil & Medicina veterinaria \\
\hline 23 & Archivos Argentinos de Pediatr & 307 & Argentina & Pediatría \\
\hline 23 & Bol Acad Nac Med Buenos Aires & 307 & Argentina & Medicina general \\
\hline 24 & Revista Brasileira Saúde Ocupac & 304 & Brasil & Salud pública \\
\hline 25 & Femina & 294 & Brasil & Ginecología \& obstetricia \\
\hline 25 & Revista Cubana de Higiene y Epi & 294 & Cuba & Salud pública \\
\hline 26 & Divulgação & 293 & Brasil & Divulgación científica \\
\hline 27 & Jornal Brasileiro de Medicina & 289 & Brasil & Medicina general \\
\hline 28 & Revista de Administração Pública & 279 & Brasil & Administración \\
\hline 39 & Acta Bioquím Clínica Latinoamer & 274 & Argentina & Bioquímica clínica \\
\hline 30 & Rev Cubana de Med Gen Integral & 273 & Cuba & Medicina general \\
\hline 31 & O Mundo da Saúde (1995) & 254 & Brasil & Medicina general \\
\hline 32 & Revista Brasileira de Oftalmolog & 253 & Brasil & Oftalmología \\
\hline 33 & Revista Argentina de Cirugía & 245 & Argentina & Cirugía \\
\hline 34 & Texto \& Contexto de Enfermagem & 241 & Brasil & Enfermería \\
\hline 35 & Revista de Microbiología & 238 & Brasil & Microbiología \\
\hline 36 & Revista Cubana de Pediatría & 236 & Cuba & Pediatría \\
\hline 37 & Arquivos Brasileiros de Oftalmol & 234 & Brasil & Oftalmología \\
\hline 38 & Ginecología y Obstetricia de Méx & 230 & México & Ginecología \& obstetricia \\
\hline 39 & Revista de Investigación Clínica & 229 & México & Investigación clínica \\
\hline 40 & Acta Paulista de Enfermagem & 225 & Brasil & Enfermería \\
\hline 40 & Arquivos Brasileiros de Cardiol & 225 & Brasil & Cardiología \\
\hline 40 & Pediatría (São Paulo) & 225 & Brasil & Pediatría \\
\hline 41 & Rev do Inst Med Trop São Paulo & 221 & Brasil & Medicina tropical \\
\hline 42 & Revista Brasileira de Odontología & 219 & Brasil & Odontología \\
\hline 43 & Revista Latino-Americ de Enferm & 217 & Brasil & Enfermería \\
\hline 44 & Boletín Epidemiol de Antioquia & 212 & Colombia & Salud pública \\
\hline 45 & Revista Chilena de Obstetr Ginec & 211 & Chile & Ginecología \& obstetricia \\
\hline 46 & Revista de Psiquiatría (Santiago) & 208 & Chile & Psiquiatría \\
\hline 47 & Revista Paulista de Enfermagem & 206 & Brasil & Enfermería \\
\hline 48 & Revista Paulista de Hospitais & 205 & Brasil & Administración \\
\hline 49 & Pediatría Moderna & 204 & Brasil & Pediatría \\
\hline 50 & Revista Chilena de Cirugía & 203 & Chile & Cirugía \\
\hline 51 & Cuadernos Médico Sociales & 202 & Argentina & Sociología médica \\
\hline 51 & Revista Cubana de Medicina & 202 & Cuba & Medicina general \\
\hline 51 & Revista do Instituto Adolfo Lutz & 202 & Brasil & Medicina general \\
\hline 52 & Ciencia e Cultura & 199 & Brasil & Divulgación científica \\
\hline 52 & Medicina y Sociedad & 199 & Argentina & Sociología médica \\
\hline 52 & Revista Cubana Aliment y Nutr & 199 & Cuba & Nutrición \\
\hline 53 & Revista Brasileira de Reumatol & 197 & Brasil & Reumatología \\
\hline 54 & Arquivos Bras Endocrinol Metab & 194 & Brasil & Endocrinología \\
\hline 55 & Gaceta Médica de Caracas & 190 & Venezuela & Medicina general \\
\hline
\end{tabular}




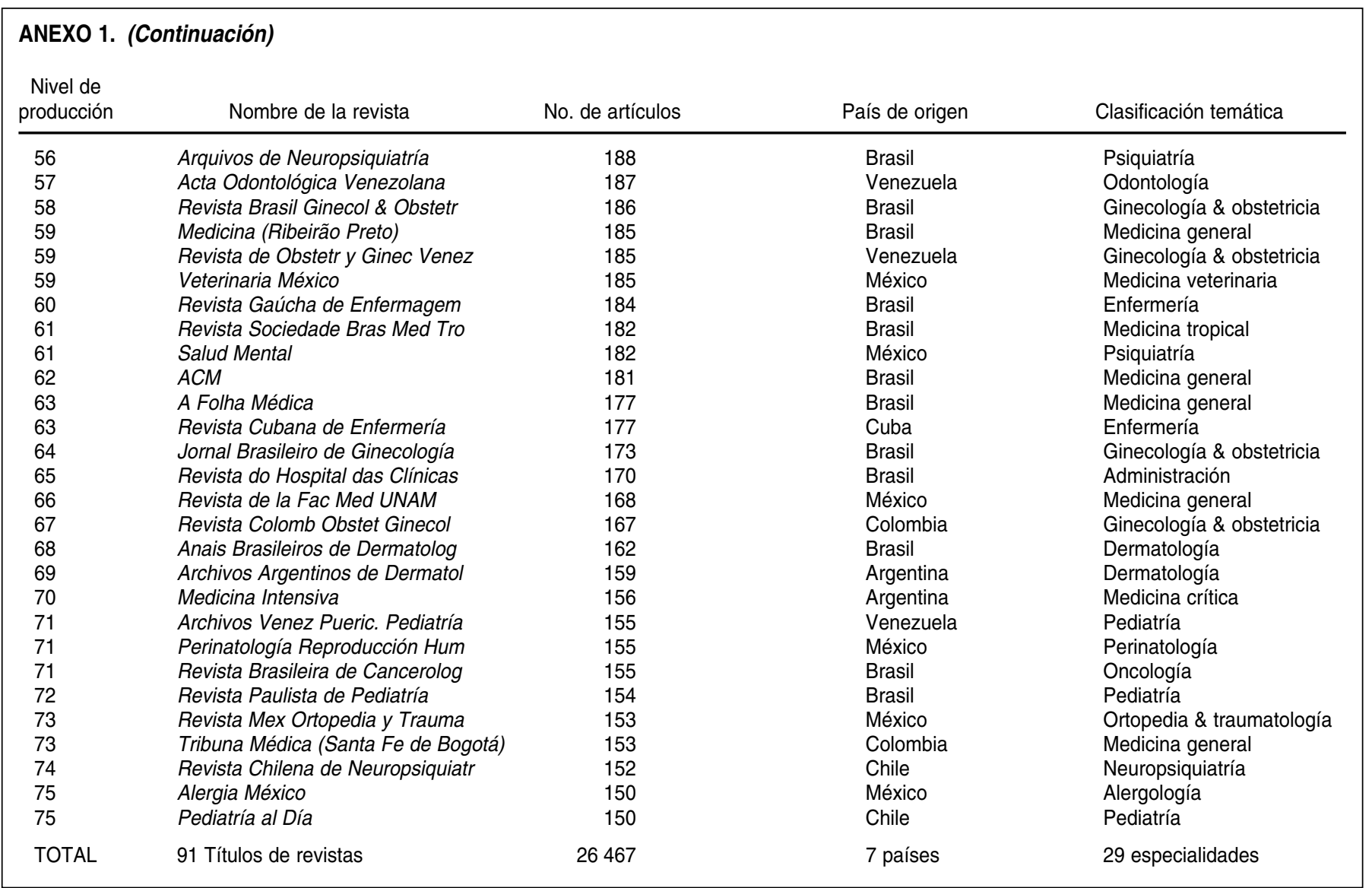

\begin{tabular}{|c|c|c|c|c|c|c|c|c|}
\hline Descriptor & BR & $\mathrm{CH}$ & $M X$ & AR & VE & $\mathrm{CO}$ & PE & $\mathrm{CU}$ \\
\hline Políticas de salud & 1 & 8 & 10 & 6 & & 8 & 12 & \\
\hline Educación de salud & 2 & & 8 & & & 14 & 11 & \\
\hline Factores socioeconómicos & 3 & 1 & 9 & & 5 & 2 & 25 & 25 \\
\hline Factores de riesgo & 4 & 2 & 1 & 1 & 6 & 1 & 7 & 1 \\
\hline Enfermería & 5 & & & & & & & \\
\hline Salud pública & 6 & 14 & 18 & & 10 & & 23 & 23 \\
\hline Servicios de salud & 7 & & 5 & & & & 3 & \\
\hline Atención de enfermería & 8 & & & & & & & 14 \\
\hline Salud mental & 9 & 10 & & 16 & & & 19 & \\
\hline Sistema de salud & 10 & & 24 & & & & & \\
\hline Cuestionario & 11 & & & & & & & \\
\hline SUS (BR) & 12 & & & & & & & \\
\hline Participación comunitaria & 13 & 25 & 20 & & & 9 & 5 & \\
\hline Vigilancia epidemiológica & 14 & & 15 & & & & & \\
\hline Salud ocupacional & 15 & & & 12 & 25 & & & \\
\hline Política social & 16 & & & & & & & \\
\hline Lactancia materna & 17 & 21 & & & & & & \\
\hline Investigación & 18 & 16 & 7 & 7 & 19 & 6 & & 15 \\
\hline Congresos & 19 & 6 & & 3 & 16 & 7 & & \\
\hline Estudios retrospectivos & 20 & 18 & & & & & 14 & 6 \\
\hline Descentralización & 21 & & & & & & & \\
\hline Estado nutricional & 22 & 5 & & & 9 & & 21 & 9 \\
\hline
\end{tabular}




\begin{tabular}{|c|c|c|c|c|c|c|c|c|}
\hline \multicolumn{9}{|l|}{ ANEXO 2. (Continuación) } \\
\hline Descriptor & BR & $\mathrm{CH}$ & $\mathrm{MX}$ & AR & VE & $\mathrm{CO}$ & PE & $\mathrm{CU}$ \\
\hline Recursos humanos de salud & 23 & & & & & & & \\
\hline Prevalencia & 24 & & & & & & 17 & \\
\hline Práctica profesional & 25 & & & & & & & \\
\hline Atención primaria de salud & & 3 & 21 & 5 & 21 & 10 & 15 & 3 \\
\hline País/ep & & 4 & 2 & & & & & \\
\hline América Latina & & 7 & & & 15 & 5 & & \\
\hline Distribución por edad & & 9 & & & & & & \\
\hline Ética médica & & 11 & & 9 & & 23 & & \\
\hline Prestación de atención de salud & & 12 & 4 & 17 & & & & \\
\hline Distribución por sexo & & 13 & & & & & & \\
\hline Morbilidad & & 15 & 16 & & & 11 & 9 & 11 \\
\hline Bioética & & 17 & 25 & & & 18 & & \\
\hline Mortalidad infantil & & 19 & 19 & 10 & & & 16 & 13 \\
\hline Calidad de vida & & 20 & & 24 & & & & \\
\hline Promoción de la salud & & 22 & 13 & & & 4 & & 16 \\
\hline Desarrollo infantil & & 23 & & & & & & \\
\hline Estudios prospectivos & & 24 & & & & & 24 & 12 \\
\hline Manuales & & & 3 & & & & & \\
\hline Indicadores de salud & & & 6 & & & 12 & & 20 \\
\hline Planes y programas de salud & & & 11 & 25 & & & & \\
\hline Mortalidad & & & 12 & & & 13 & 13 & \\
\hline Programas nacionales de salud & & & 14 & & & & & 4 \\
\hline Planificación familiar & & & 17 & & & 15 & & \\
\hline Calidad de la atención de salud & & & 22 & 15 & & & & \\
\hline Encuestas epidemiológicas & & & 23 & & & & & 22 \\
\hline Odontólogos & & & & 2 & & & & \\
\hline Médicos & & & & 4 & & & & \\
\hline Neoplasias & & & & 8 & & & & \\
\hline Seguridad social & & & & 11 & & & & \\
\hline Control de calidad & & & & 13 & & & & \\
\hline Relaciones médico-paciente & & & & 14 & & & & \\
\hline Enfermedades de la piel & & & & 18 & & & & \\
\hline Salud & & & & 19 & & & & \\
\hline Práctica profesional & & & & 20 & & & & \\
\hline Responsabilidad legal & & & & 21 & & & & \\
\hline Audición & & & & 22 & & & & \\
\hline Ortopedia & & & & 23 & & & & \\
\hline Medicina & & & & & 1 & & & \\
\hline Nutrición & & & & & 2 & & 10 & \\
\hline Valor nutritivo & & & & & 3 & & & \\
\hline Dieta & & & & & 4 & & & \\
\hline Pediatría & & & & & 7 & & & \\
\hline Manipulación de alimentos & & & & & 8 & & & \\
\hline Niño & & & & & 11 & & & \\
\hline Fabaceae & & & & & 12 & & & \\
\hline Crecimiento & & & & & 13 & & & \\
\hline Peso corporal & & & & & 14 & & & 7 \\
\hline Alimentos fortificados & & & & & 17 & & & \\
\hline Brasil & & & & & 18 & & & \\
\hline Antropometría & & & & & 20 & & & 19 \\
\hline Chile & & & & & 22 & & & \\
\hline Hábitos alimentarios & & & & & 23 & & & \\
\hline Necesidades nutricionales & & & & & 24 & & & \\
\hline Derechos humanos & & & & & & 3 & & \\
\hline Erupciones volcánicas & & & & & & 16 & & \\
\hline Planificación en desastres & & & & & & 17 & & \\
\hline Planificación hospitalaria & & & & & & 19 & & \\
\hline Conocimientos, actitudes y prácticas & & & & & & 20 & 4 & \\
\hline Investigación/ME & & & & & & 21 & & \\
\hline Estado de salud & & & & & & 22 & & 8 \\
\hline Desastres naturales & & & & & & 24 & 22 & \\
\hline Efectos de desastres en la salud & & & & & & 25 & & \\
\hline Salud ambiental & & & & & & & 1 & \\
\hline Epidemiología descriptiva & & & & & & & 2 & 5 \\
\hline Estudios transversales & & & & & & & 6 & 24 \\
\hline
\end{tabular}




\begin{tabular}{|c|c|c|c|c|c|c|c|c|}
\hline \multicolumn{9}{|l|}{ ANEXO 2. (Continuación) } \\
\hline Descriptor & $\mathrm{BR}$ & $\mathrm{CH}$ & $\mathrm{MX}$ & $A R$ & VE & $\mathrm{CO}$ & $\mathrm{PE}$ & $\mathrm{CU}$ \\
\hline Altitud & & & & & & & 8 & \\
\hline Salud maternoinfantil & & & & & & & 18 & \\
\hline Abastecimiento de agua & & & & & & & 20 & \\
\hline Médicos de familia & & & & & & & & 2 \\
\hline Estatura & & & & & & & & 10 \\
\hline Unidades de terapia intensiva & & & & & & & & 17 \\
\hline Grosor de pliegues cutáneos & & & & & & & & 18 \\
\hline Recién nacido de peso bajo & & & & & & & & 21 \\
\hline
\end{tabular}

\section{REFERENCIAS}

1. Frenk J, Bobadilla JL, Sepúlveda J, Recental J, Ruelas E. Un modelo conceptual para la investigación en salud pública. Bol Of Sanit Panam. 1986;101(5):477-89.

2. White F. Capacity-building for health research in developing countries: a manager's approach. Rev Panam Salud Publica. 2002; 12(3):165-71.

3. Horton R. The health (and wealth) of nations. Lancet. 2002;359(9311):993-4.

4. González García G. Las reformas sanitarias y los modelos de gestión. Rev Panam Salud Publica. 2001;9(6):406-12.

5. Londono JL, Frenk J. Structured pluralism: towards an innovative model for health system reform in Latin America. Health Policy. 1997; 41(1):1-36.

6. Global Forum on Health Research. The 10/90 report on health research 1999. Geneve: World Health Organisation, 1999.

7. Lancet Editorial. Global health needs a new research agenda. The Lancet. 2004;364(9445): $1555-6$.

8. Arunachalam S, Gunasekaran S. Tuberculosis research in India and China: from bibliometrics to research policy. Curr Sci. 2002;82(8): 933-47.

9. Arunachalam S, Gunasekaran S. Diabetes research in India and China today: from literature-based mapping to health-care policy. Curr Sci. 2002;82(9):1086-97.

10. Lewison G, Henderson C, Willcox-Jay K, Barnes S, Chilvers C. The anatomy of mental health research in 15 OECD countries. Paper presented at the Science and Technology Indicators Conference; 2002; Karlsruhe, Germany.

11. San-Blas G. From magic to science: a journey throughout Latin America medical Mycology. Med Mycol. 2000;38 (Suppl 1):1-8.

12. Mas-Castella J. 4 years of Microbiologia SEM (1994-1997). Microbiologia. 1997;13(4):50916.

13. Figuera LE, Cantu JM. The contribution of Latin America to knowledge of human genome. Bol Oficina Sanit Panam. 1993;115(1): $12-8$.

14. Weisinger JR, Vellori-Font E. Latin American Nephrology: scientific production and impact of publications. Kidney Int. 1999;56(4):158490.

15. Rodrigues PS, Fonseca L, Chaimovich $\mathrm{H}$. Mapping cancer, cardiovascular and malaria research in Brazil. Braz J Med Biol Res. 2000; 33(8):853-67.
16. Langer A. International cooperation for research in public health: the case of the Latin American Network of Perinatal Investigation. Salud Publica Mex. 1991;33(4):410-6.

17. Macías-Chapula CA. Patterns of scientific communication among Latin American countries in the field of medical education. Scientometrics. 1992;23(1):123-35.

18. Macías-Chapula CA. Análisis informétrico de la producción científica y técnica en México sobre contaminación ambiental y salud. REDIAL. 1994;4:71-6.

19. Macías-Chapula C.A. Primary health care in México: a non-ISI bibliometric analysis. Scientometrics. 1995;34(1):63-71.

20. Macías-Chapula CA, Rodea-Castro IP. Subject content of the Mexican production on health and the environment (1982-1993). Scientometrics. 1997;38(2):295-308.

21. Macías-Chapula CA, Rodea-Castro IP, Narváez-Berthelemot N. Bibliometric analysis of AIDS literature in Latin America and the Caribbean. Scientometrics. 1998;41(1-2):41-9.

22. Macías-Chapula CA, Sotolongo-Aguilar GR, Madge B, Solorio-Lagunas J. Subject content analysis of AIDS literature, as produced in Latin America and the Caribbean. Scientometrics. 1999;46(3):563-74.

23. Macías-Chapula CA. AIDS in Haiti: a bibliometric analysis. Bull Med Libr Assoc. 2000; 88(1):56-61.

24. Tapia L, Torrente M, Posada C. Authorship in the chilean journal Revista Otorrinolaringología y Cirugía de Cabeza y Cuello: analysis of three decades. Rev Med Chil. 2002; 130(7):773-8.

25. Pasqualini CD, Kotsias BA. Past and present of Medicina (Buenos Aires). Medicina (B Aires). 2000;60(1):1-7.

26. Krauskopf M. A scientometric radiography of Revista Médica de Chile. Rev Med Chil. 1997; 125(7):775-80.

27. Macías-Chapula CA. Análisis de citas de cuatro revistas biomédicas latinoamericanas. Rev Esp Doc Cient. 1991;14(4)420-7.

28. Pellegrini Filho A, Goldbaum M, Silvi J. Production of scientific articles about health in six Latin American countries, 1973-1992. Rev Panam Salud Publica. 1997;1(1):23-4.

29. Roselli D. Latin American biomedical publications: the case of Colombia in Medline. Med Educ. 1998;32(3):274-7.

30. Lotufo PA. Medicine based in Brazilians. Arq Bras Cardiol. 2001;76(5):419-20.
31. Rodríguez Lamoine V. Scientific publication in the Andean subregion. Acta Cient Venez. 1992;43(6):321-2.

32. Ganz Brown C, Coward R, Stowe R. Assessing the scientific strength of Chile. Arch Biol Med Exp. 1991;24(1):37-47.

33. Valdez Martínez E. Análisis bibliométrico de las publicaciones realizadas por el personal del IMSS. Gac Med Méx. 2000;136(4):319-28.

34. Allende JE. Latin American science from the clinical perspective. Microbiologia. 1997;13(2): 127-30.

35. Ayala FJ. Science in Latin America. Microbiologia. 1996;12(2):163-6.

36. Macías-Chapula CA. Non-SCI subject visibility of the Latin American scientific production in the health field. Scientometrics. 1994;30(1): 97-104.

37. Sandoval A, Núñez A. Publicación fuera de la región de artículos por especialistas latinoamericanos sobre cuestiones biomédicas. Bol UNESCO. 1974;28(1):1-6.

38. Bracho-Riquelme RL, Pescador-Salas N, Reyes-Romero MA. Repercusión bibliométrica de adoptar el Inglés como idioma único de publicación. Rev Invest Clin. 1997;49(5): 369-72.

39. Macías-Chapula CA. Bibliometric and webometric analysis of health system reforms in Latin America and the Caribbean. Scientometrics. 2002;53(3):407-27.

40. Garfield E. Quantitative analysis of the scientific literature and its implications for science policy making in Latin America and the Caribbean. Bol Of Sanit Panam. 1995;29(1):87-95.

41. Coimbra CE Jr. Scientific production in public health and the international bibliographic databases. Cad Saude Publica. 1999;15(4): 883-8.

42. Narro-Robles JR, Gutiérrez-Avila JH. The development of public health research. Salud Publica Mex. 1991;33(6):605-16.

43. Porta M. The bibliographic impact factor of the Institute for Scientific Information: How relevant is it really for public health journals? J Epidemiol Community Health. 1996;50(6): 606-10.

44. de los Ríos R. Promotion of public health research: attempting to balance relevance and excellence. Rev Panam Salud Publica. 1999; 5(4-5):309-15.

45. OPS. Producción científica en salud 19791988. Washington: Organización Panamericana de la Salud; 1989. 
46. Almada de Ascencio M. Tendencias de la producción científica en cinco países de América Latina. En: Lezana MA, Faba G. La producción científica en salud en México. México: Secretaría de Salud; 1992; 85-109.

47. Pellegrini Filho A. El proyecto regional sobre la situación de la investigación en salud en América Latina. En: Lezana MA y Faba G. La producción científica en salud en México; México: Secretaría de Salud; 1992;19-30.

48. Pellegrini Filho A. Estado de avance del proyecto regional. En: Lezana MA, Faba G. La Producción Científica en Salud en México. México: Secretaría de Salud; 1992;115-51.
49. Gibbons M. Science's new social contract with society. Nature. 1999;402(Suppl):c82-c84.

50. Macías-Chapula CA. Estudio explorativo sobre un modelo de comunicación de la ciencia en el área de la salud. En: Almada de Ascencio M, Liberman Sofía y Russell JM. Investigación sobre la comunicación científica: un enfoque multidisciplinario. México: Universidad Nacional Autónoma de México; 2002; 55-68.

51. Macías-Chapula CA. Análisis bibliométrico de la producción científica sobre salud pública en América Latina y el Caribe (1980-
2002). Reporte Técnico. Hospital General de México. México DF, mayo de 2003.

52. Bradford SC. Documentation. Londres: Crosby Lockwood; 1948

53. Bookstein A. Towards a multidisciplinary Bradford law. Scientometrics. 1994;30(1): 353-61.

Manuscrito recibido el 29 de diciembre de 2004. Aceptado para publicación, tras revisión, el 30 de agosto de 2005.
ABSTRACT

Toward a model of communications in public health in Latin America and the Caribbean
Objective. So far, there have been no bibliometric or scientometric studies that make it possible to examine, with quantitative, retrospective, and comprehensive criteria, the scientific output on public health in Latin America and the Caribbean (LAC). Further, the weakness of the existing information systems makes it impossible to examine the relevance, quality, and impact of this scientific output, with a view to evaluating it in terms of societal needs and existing patterns of scientific communication. This article presents the results of a bibliographic analysis of the scientific output in the area of public health in Latin America and the Caribbean. The ultimate goal of the analysis is to build a model of scientific communication in this field, to help researchers, managers, and others working in the area of public health to make decisions and choose actions to take.

Methods. We conducted a literature review in order to identify the distribution of publications on public health that were produced by LAC researchers and published in each of the LAC countries from 1980 through 2002. The review used the Literatura Latino-Americana e do Caribe em Saúde Pública (LILACS-SP) (Latin American and Caribbean Literature on Public Health) bibliographic database. That database is operated by the Latin American and Caribbean Center on Health Sciences Information (BIREME), which is in São Paulo, Brazil. We processed the LILACS-SP data using two software packages, Microsoft Excel and Bibexcel, to obtain indicators of the scientific output, the type of document, the language, the number of authors for each publication, the thematic content, and the participating institutions. For the 1980-2002 period, there were 97605 publications registered, from a total of 37 LAC countries.

Results. For the analysis presented in this article, we limited the sample to the 8 countries in Latin America and the Caribbean that had at least 3000 documents each registered in the LILACS-SP database over the 1980-2002 study period. In descending order of the number of publications registered, the 8 nations were: Argentina, Brazil, Chile, Colombia, Cuba, Mexico, Peru, and Venezuela. Those 8 countries were responsible for 83054 publications $(85.10 \%$ of the total of 97605 registered documents produced by the 37 LAC countries). Of those 83054 publications from the 8 countries, 56 253 of them $(67.73 \%)$ were articles published in scientific journals and 24488 were monographs $(29.48 \%)$. The proportion of works produced by two or more coauthors was relatively high (56.48\%). The 56253 articles appeared in a total of 929 different journals. Of the 929 journals, 91 of them published at least 150 articles over the study period. In descending order, LAC journals with the largest number of articles on public health were: Revista de Saúde Pública (Brazil); Cadernos de Saúde Pública (Brazil); Revista Médica de Chile; Archivos Latinoamericanos de Nutrición (Venezuela); and Salud Pública de México. The 91 journals that published at least 150 articles represented 29 different specialties. The most common of the specialties for the 91 journals were general medicine (18 journals) and pediatrics (10 journals). In descending order, the populations that the publications dealt with primarily were human beings in general, females, males, and adults; and, in descending order, a relatively small number of publications dealt with pregnant women and middle-aged or elderly persons. The topics most often covered in the publications were risk factors, health policy, and primary health care, as well as family doctors in the case of Cuba.

Conclusions. This research produced a preliminary model of communications in public health in LAC countries that will hopefully help lay the groundwork for further research to develop a model of scientific communication in LAC nations.

Key words Public health, research, publications, periodicals, bibliometrics, Latin America, Caribbean region. 